follow-up (RD: 0.28; 95\% CI: -0.03-0.75). Xiao Pi Kuan Wei Decoction may have a large beneficial effect on alleviating postprandial fullness (Standardised mean difference (SMD): 2.14; 95\% CI: $-2.76-0.70$ ), early satiety (SMD: $-3.90 ; 95 \%$ CI: $-0.68--0.42$ ), and epigastric pain (SMD: -1.23 ; 95\% CI: -1.66- -0.29). No serious adverse events were reported.

Conclusions Modified Zhi Zhu Decoction and Xiao Pi Kuan Wei Decoction may be considered as an alternative for patients unresponsive to prokinetics. Confirmatory head-tohead trials should be conducted to investigate their comparative effectiveness against prokinetics.

\section{IDDF2021-ABS-0056 LIVER STIFFNESS PLUS PLATELETS PREDICTS LONG-TERM OUTCOMES IN CHILD-PUGH A CIRRHOSIS}

${ }^{1}$ Tanya Lee* ${ }^{2}$ Elliot Freeman, ${ }^{2}$ Daniel Clayton-Chub, ${ }^{2}$ Stuart Roberts, ${ }^{1}$ Nik Ding, ${ }^{2}$ William Kemp, ${ }^{2}$ Rohit Sawhney, ${ }^{2} J o h n$ Lubel, ${ }^{1}$ David Iser, ${ }^{1}$ Jessica Howell, ${ }^{1}$ Catherine Croagh, ${ }^{1}$ Thai Hong, 'Barbara Demediuk, ${ }^{1}$ Paul Desmond, ' 5 wee Lin Chen Yi Mei, ${ }^{1}$ Alexander Thompson, ${ }^{1}$ Jacinta Holmes. 'St Vincent's Hospital, Australia; ${ }^{2}$ Alfred Health, Australia

\subsection{6/gutjnl-2021-IDDF.74}

Background Portal hypertension (PH) remains an important sequela of cirrhosis, contributing to morbidity and mortality. Non-invasive algorithms identifying patients at risk of clinically significant portal hypertension (CSPH) and high-risk gastrooesophageal varices (varices needing treatment, VNT) are critical. We previously described an algorithm whereby liver stiffness measurement (LSM) $\leq 25 \mathrm{kPa}$ plus platelets $\geq 100$ excluded VNT in Child-Pugh A cirrhosis. 5 years following the inception of our initial study, this study aimed to assess the prognostic value of $\mathrm{LSM} \leq 25 \mathrm{kPa}$ plus platelets $\geq 100$ in predicting long-term hepatic decompensation and death.

Methods This is a retrospective multicentre study of patients from two tertiary centres. Medical records were reviewed for all subsequent gastroscopies, LSM, six-monthly laboratory tests and abdominal ultrasounds, symptoms/signs consistent with hepatic decompensation (ascites, variceal bleeding, hepatic encephalopathy), and mortality from the initial study date. Patients with an LSM $>25 \mathrm{kPa}$ plus platelets $<100$ were considered high risk for VNT, while patients with $\mathrm{LSM} \leq 25 \mathrm{kPa}$ and/or platelets $\geq 100$ were considered low risk.

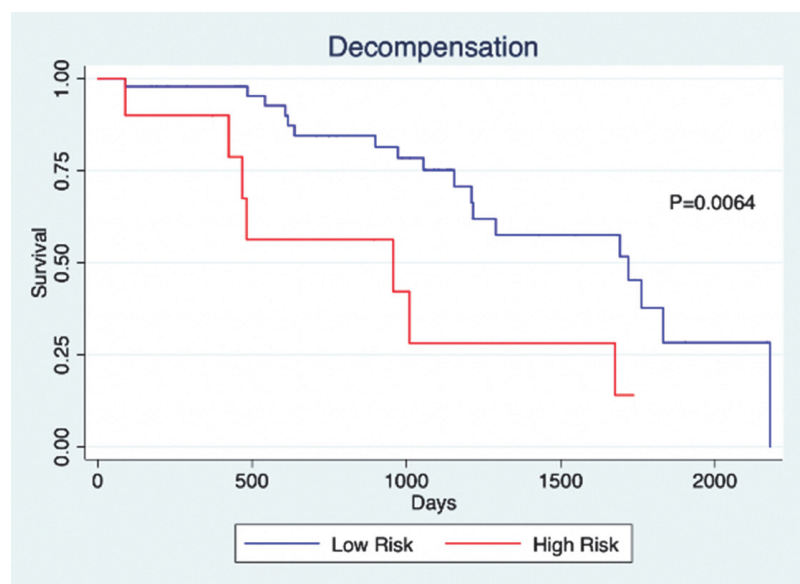

Abstract IDDF2021-ABS-0056 Figure 1

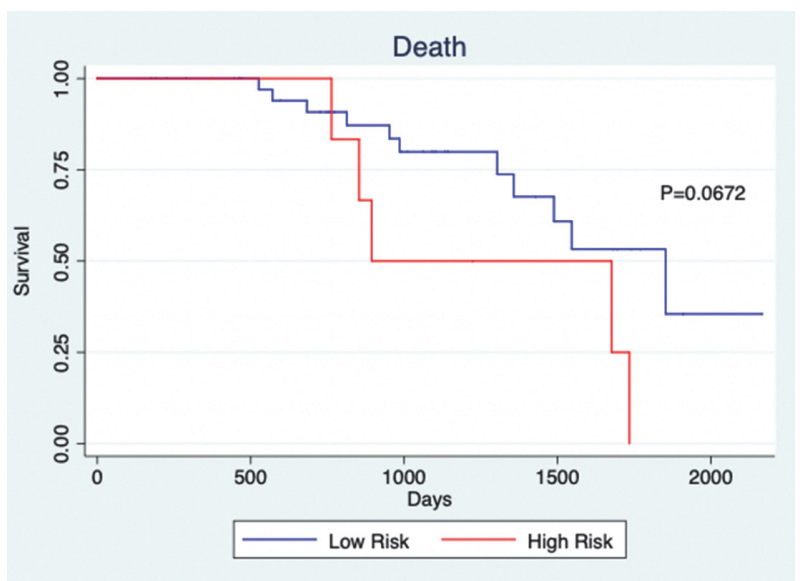

Abstract IDDF2021-ABS-0056 Figure 2

Results 180 patients were included. Median age was 55 yrs (IQR 49-63), 70\% male, with no significant difference between groups. Median follow-up was 7.7 yrs (4.5-9.0). At study inception, 30/180 (17\%) were high risk. The majority $(114 / 180,63 \%)$ had cirrhosis secondary to HCV, similar according to risk group $(\mathrm{p}=0.752)$. Decompensation was significantly more common in high-risk patients (11/30 (37\%) vs 26/150 (17\%) respectively, $p=0.016$; (IDDF2021-ABS-0056 Figure 1. Decompensation according to high-risk and low-risk category)). 2/150 (1\%) low-risk patients were re-classified as high-risk. 19/150 (13\%) of low-risk patients had VNT compared to $7 / 30(23 \%)$ in the high-risk group $(p=0.043)$. There was a trend towards higher mortality in high-risk vs low-risk patients $(12 / 30(40 \%)$ vs $36 / 150$ (24\%), respectively, $p=0.067$; (IDDF2021-ABS-0056 Figure 2. Death according to high-risk and low-risk category)).

Conclusions Our previously defined algorithm of LSM and platelets has prognostic value in predicting for subsequent decompensation and death. LSM+PLT can assist in stratifying future risk of liver-related events in Child-Pugh A cirrhotics.

\section{IDDF2021-ABS-0064 ASCITIC FLUID TOTAL CELL COUNT AND POLYMORPHONUCLEAR CELL COUNT IN PATIENTS WITH CIRRHOSIS AND ASCITES AND ITS PROGNOSTIC VALUE}

Parimita Barua, Krishnasamy Narayanasamy*. Madras Medical College, India

\subsection{6/gutjnl-2021-IDDF.75}

Background Spontaneous bacterial peritonitis (SBP) is defined as ascitic fluid (AF) infection without a surgically treatable intra-abdominal source of infection with or without a positive $\mathrm{AF}$ culture and $\mathrm{AF}$ polymorphonuclear(PMN)cell count of $>250$. In this study, we assessed the prognostic value of $\mathrm{AF}$ total cell count and PMN cell count in patients with cirrhosis and ascites and its outcome.

Methods The prospective study included patients with cirrhosis and ascites. Patients biochemical parameters, manual ascitic fluid total cell and PMN count were studied. Based on AF PMN, patients were categorised into, Reactive effusion (RE)AF $\mathrm{PMN}<150$, Neutrophillic effusion(NE)AF PMN 150-250 and SBP-AF PMN $>250$. Patients were followed up at the end of 1 and 6 months to study the outcome (survival/expired). 